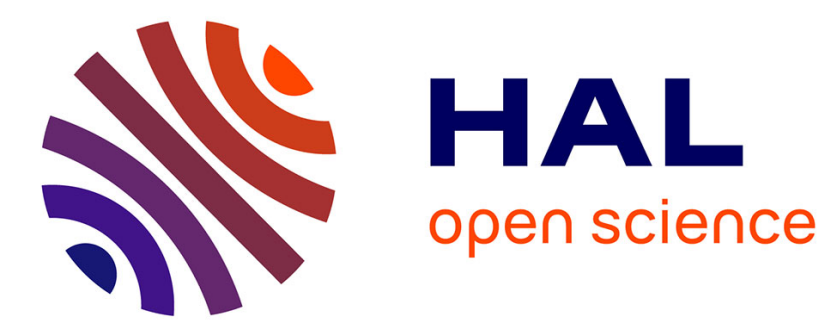

\title{
Soft laser sputtering of GaAs semiconductor surfaces
}

\author{
L. Vivet, M. Barthe, T. Gibert, B. Dubreuil
}

\section{To cite this version:}

L. Vivet, M. Barthe, T. Gibert, B. Dubreuil. Soft laser sputtering of GaAs semiconductor surfaces. Journal de Physique IV Proceedings, 1994, 04 (C4), pp.C4-115-C4-118. 10.1051/jp4:1994424 jpa00252687

\section{HAL Id: jpa-00252687 https://hal.science/jpa-00252687}

Submitted on 1 Jan 1994

HAL is a multi-disciplinary open access archive for the deposit and dissemination of scientific research documents, whether they are published or not. The documents may come from teaching and research institutions in France or abroad, or from public or private research centers.
L'archive ouverte pluridisciplinaire HAL, est destinée au dépôt et à la diffusion de documents scientifiques de niveau recherche, publiés ou non, émanant des établissements d'enseignement et de recherche français ou étrangers, des laboratoires publics ou privés. 


\title{
Soft laser sputtering of GaAs semiconductor surfaces
}

\author{
L. VIVET, M.F. BARTHE*, T. GIBERT and B. DUBREUIL
}

GREMI, CNRS, Université d'Orléans, BP. 6759, 45067 Orléans cedex 2, France

* CERI, CNRS, Orléans, France

\section{INTRODUCTION}

Laser sputtering of III-V compound semiconductors is currently the subject of experimental and theoretical investigations for potential applications in optoelectronics (processing or improvement of devices, production of original surface structures). Elemental analysis of doping or impurity concentrations in GaAs/GaAlAs multilayer structures with depth resolution of a few nanometers, which also minimises mixing at the interfaces is an important challenge. For this purpose, sputtering with low fluence UV laser is regarded as an interesting alternative to the conventional ion beam sputtering method.

We have studied the soft laser sputtering of GaAs surfaces with $337 \mathrm{~nm}$ photons, starting from the threshold for particle emission (a few tens $\mathrm{mJ} / \mathrm{cm}^{2}$ ) up to $220 \mathrm{~mJ} / \mathrm{cm}^{2}$ fluences. Particles (atoms and molecules) sputtered from the irradiated surface are detected, their relative number measured and their time-of-flight determined using laser resonant ionisation mass spectrometry (RIMS). The surface state after laser irradiation is examined by SEM and $X$ analysis.

\section{2 - EXPERIMENTAL STUDY}

The experimental set-up described in details in Ref.1 is mainly composed of an ultra-high vacuum chamber equipped with a sample manipulator and a quadrupole massspectrometer. The GaAs sample is irradiated by the beam of a $\mathrm{N}_{2}$ laser $(337 \mathrm{~nm}$ ) of $10 \mathrm{~ns}$ pulse duration and $10 \mathrm{~Hz}$ repetition rate. After aperturing and focusing on the sample with an $\mathrm{f}=30 \mathrm{~cm}$ lens at a $60^{\circ}$ incidence angle, the maximum fluence is about $220 \mathrm{~mJ} / \mathrm{cm}^{2}$. Atomic and molecular sputtered species are detected by the mass-spectrometer, directly in the case of ionized particles and after resonant multiphoton ionization by the second harmonic of a tunable Nd: Yag pumped dye laser for the neutrals. This probe laser pulse is fixed with an adjustable delay ( 0 to $14 \mu \mathrm{s}$ ) with respect to the $\mathrm{N}_{2}$ laser pulse.

\subsection{Photoionization spectrum of Ga and As species}

$\mathrm{Ga}^{\circ}$ atoms are probed in their ${ }^{2} \mathrm{P}_{1 / 2}^{o}$ ground state by $(1+1)$ resonant multiphoton ionization at $\lambda=287.5 \mathrm{~nm}$, whereas ionization of $\mathrm{As}^{\circ}$ atoms requires a $(2+1)$ multiphoton process at $\lambda=286 \mathrm{~nm}\left({ }^{4} S_{3 / 2}^{\circ} \rightarrow{ }^{4} D_{1 / 2,3 / 2} 2\right.$-photon resonances). In fact, scanning the probe laser wavelength around these two resonances, we observe the spectrum shown in Fig.1. It is composed of three lines, the unexpected broader line being attributed to a $(1+1)$ photon transition at $\lambda=286.127 \mathrm{~nm}$ starting from the ${ }^{2} \mathrm{P}_{3 / 2}^{\circ}$ excited state $\left(18186 \mathrm{~cm}-{ }^{1}\right)$. It will be shown in the following that this spectrum results from the photodissociation of $\mathrm{As}_{2}$ molecules which constitute the largest part of the sputtered arsenic species. According to this result, the photoionization schemes leading to the observed spectrum are: 


$$
\begin{aligned}
A s_{2}+h v & \rightarrow A s\left({ }^{2} P_{3 / 2}^{o}\right)+h v \rightarrow A s\left({ }^{2} P_{1 / 2}\right)+h v \rightarrow A s^{+} \text {line } \mathrm{n}^{\circ} 2 \\
& \rightarrow A s\left({ }^{4} S_{3 / 2}^{o}\right)+2 h v \rightarrow A s\left({ }^{4} D_{3 / 2,1 / 2}^{o}\right)+h v \rightarrow A s^{+} \text {lines } \mathrm{n}^{\circ} 1 \text { and } 3
\end{aligned}
$$

A part of $A s_{2}$ molecules are also photoionized without dissociation by three-photon absorption. The spectrum is relatively flat in the 287 - $290 \mathrm{~nm}$ range. On the contrary, the $\mathrm{Ga}_{2}$ photoionization spectrum presents structures characteristic of rovibrational bands.

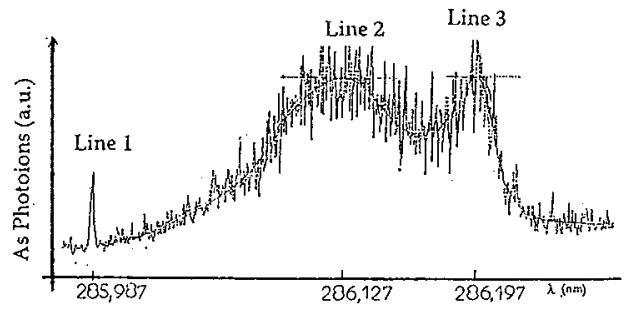

Fig. 1- Photoionisation spectrum of laser -sputtered arsenic

\subsection{Time of flight distributions}

Time of flight (TOF) distributions of $\mathrm{Ga}^{\circ}, \mathrm{As}^{\circ}$ and $\mathrm{As}_{2}{ }^{\circ}$ species were measured by delaying the probe laser pulses with respect to the $\mathrm{N}_{2}$ laser shots. The results shown in Fig. 2 correspond to $170 \mathrm{~mJ} / \mathrm{cm}^{2} \mathrm{~N}_{2}$ laser fluence and a probing distance of $3.6 \mathrm{~mm}$ above the sample surface. One observes that $\mathrm{As}^{\circ}$ and $\mathrm{As}_{2}{ }^{\circ}$ species have the same TOF. This proves that the observed $A s^{\circ}$ photoions originate mainly from the photodissociation of $A s^{\circ}{ }_{2}$ molecules, i.e. arsenic is mainly sputtered by laser irradiation in the form of diatomic species and not as atoms, contrary to gallium.

The TOF distributions of $\mathrm{Ga}^{\circ}$ atoms and $\left(\mathrm{As}^{\circ}\right) \mathrm{As}_{2}^{\circ}$ molecules are well fitted by half-space Maxwellian distributions. characterized by kinetic temperatures. ${ }^{1}$ They are in broad agreement with the results obtained from a model of laser heating of the surface. In the conditions of soft laser sputtering, the energy of the sputtered particles reflects the "thermal" state of the surface. The particles temperature as well as the surface temperature increase with the laser fluence, reaching the melting temperature of GaAs $(1500 \mathrm{~K})$ for 200 $\mathrm{mJ} / \mathrm{cm}^{2}$.

\subsection{Laser Sputtering Yields}

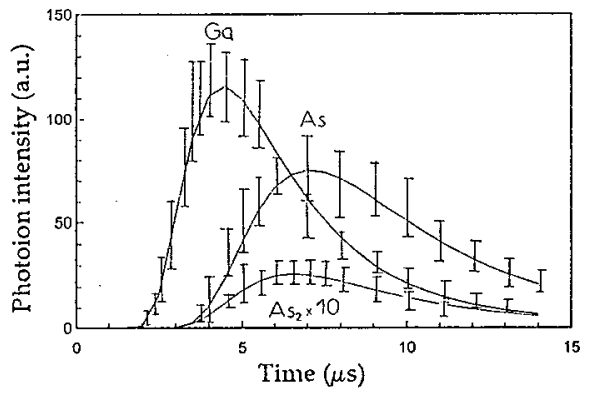

Fig. 2 - TOF distribution of Ga and As species Fit by Maxwellian functions with $\mathrm{T}=1350 \mathrm{~K}$

a- shot number dependencies

The variation of the sputtering yield with the number of laser shots on the same spot is shown in Fig. 3 for $\mathrm{Ga}^{\circ}$ and $\left(\mathrm{As}^{\circ}\right) \mathrm{As}_{2}^{\circ}$ for different laser energies. During the first laser shots, one observes a very large emission of particles, followed by a fast decrease. After one thousand laser shots, the signal is decreased by a factor of 2 for $\mathrm{Ga}^{\circ}$ and up to 10 for $\left(A s^{\circ}\right) A s_{2}^{\circ}$. Then, the yields decrease slowly and tend towards a quasi stationary regime for a number of shots greater than $10^{4}$. This behaviour is roughly observed whatever the laser fluence is. However, one must distinguished two fluence ranges.

For low laser values (from the threshold to $85 \mathrm{~mJ} / \mathrm{cm}^{2}$ ), As emission vanishes after a few thousand laser shots. This can be interpreted as the sputtering of the surface defects resulting from localized absorption on the defect sites followed by bond breaking. ${ }^{2}$ It must 
be noted that in this low fluence range, the kinetic temperature of the sputtered particles as well as the calculated surface temperature are less than $800 \mathrm{~K}$, that is much lower than the melting temperature of $\mathrm{GaAs}(1500 \mathrm{~K})$ and the critical temperature for strong $\mathrm{As}_{2}$ vaporisation $(950 \mathrm{~K}){ }^{3}$ In the medium fluence range $\left(85-220 \mathrm{~mJ} / \mathrm{cm}^{2}\right)$, the sputtering yields tend towards a near constant value after a large number of shots. However, a fine examination of the $\mathrm{Ga} / \mathrm{As}$ sputtering yield ratio shows that the composition of the irradiated zone evolves as the shot number increases : the ratio increases due to the slow but continuous As-impoverishment of the irradiated surface.
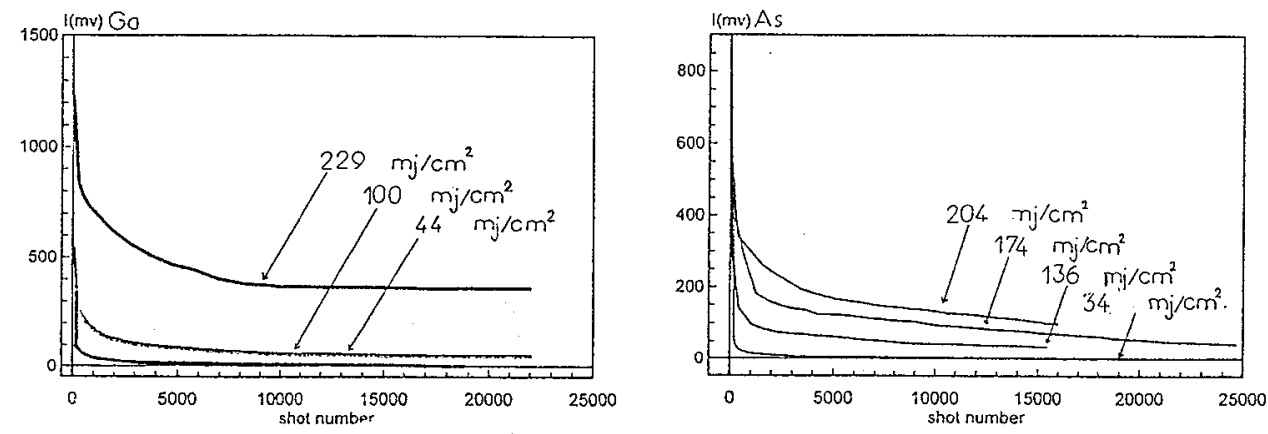

Fig.3 - Laser sputtering yields of Ga and As as a function of the number of laser shots

This behaviour is correlated to the strong emission of As species in the form of $\mathrm{As}_{2}$ molecules at the beginning of the sputtering. The preferential sputtering of arsenic leads to the formation of a perturbed Ga-rich structure after a few laser shots. This initial transformation seems to determine the further evolution of the irradiated surface in an irreversible way. Contrary to the case of InP where preferential sputtering of $\mathrm{P}$ was also observed, ${ }^{4}$ no compositional equilibrium of the altered surface is reached for GaAs. This is probably due to the particular thermodynamical properties of this material which incongruently evaporates for temperature larger than $950 \mathrm{~K}$. For example, at $1300 \mathrm{~K}$, the partial pressure of $A s_{2}$ and $A s_{4}$ is more than 2 orders of magnitude larger than for $\mathrm{Ga}$. According to our TOF measurements and the calculations, this surface temperature should be obtained for only $\sim 170 \mathrm{~mJ} / \mathrm{cm}^{2}$ laser fluence.

\section{4- Scanning electron microscopy and $X$ analysis of the irradiated spots}

The above discussion related to preferential sputtering of $\mathrm{As}_{2}$ and the consecutive formation of a perturbed Ga-rich surface is illustrated by the microscopic analysis of the structural and compositional changes of the irradiated spots, for different numbers of laser shots. For example, Fig. 4 reproduces an image of the surface irradiated by $2 \times 10^{5} 220$ $\mathrm{mJ} / \mathrm{cm}^{2}$ laser shots. $X$ analysis of the As and Ga contents are given for different positions in the perturbed zone. Different structures are observed in the form of droplets, flakes and piles. All these structures show a large reduction of the As concentration. One observes also that this reduction is the largest at the spot center, according to the laser energy distribution. But, the compositions of the spot center and droplets surrounding the spot are quite similar : the As / Ga ratio is reduced to 0.16.

The formation of Ga-rich droplets is also observed for low laser fluence (40 $\left.\mathrm{mJ} / \mathrm{cm}^{2}\right)$ but after $710^{4}$ laser shots and for moderate fluence $\left(125 \mathrm{~mJ} / \mathrm{cm}^{2}\right)$ after only $210^{4}$ shots. In Ref. 5, similar production of Ga clusters after $5 \mathrm{~mm}$ thermal annealing of a GaAs surface at $930 \mathrm{~K}$ was reported. By analogy, we might consider that the laser pulse acts as a pulsed heating source increasing the surface temperature. According to the particular phase 
diagram of GaAs, when exceeding a critical value, this temperature increase leads to the preferential emission of arsenic in the form of molecules. This induces the formation of a Garich surface structure. In the case of low laser fluence (i.e. below the critical temperature), the process could be initiated from the heating of surface defects which are weakly bound to the surface. In both cases, this initial transformation seems to determine the further evolution of the irradiated surface. Following $\mathrm{As}_{2}$ emission, unbounded $\mathrm{Ga}$ atoms aggregates to form $\mathrm{Ga}$ islands on the surface. After a sufficient number of shots, micrometric structures are produced which finally behave as the pure Ga metal. This evolution of the surface state after multipulse laser irradiation appears to be practically the same for low and medium laser energies, the only difference being in the number of shots required to obtain the same microscopic structure.

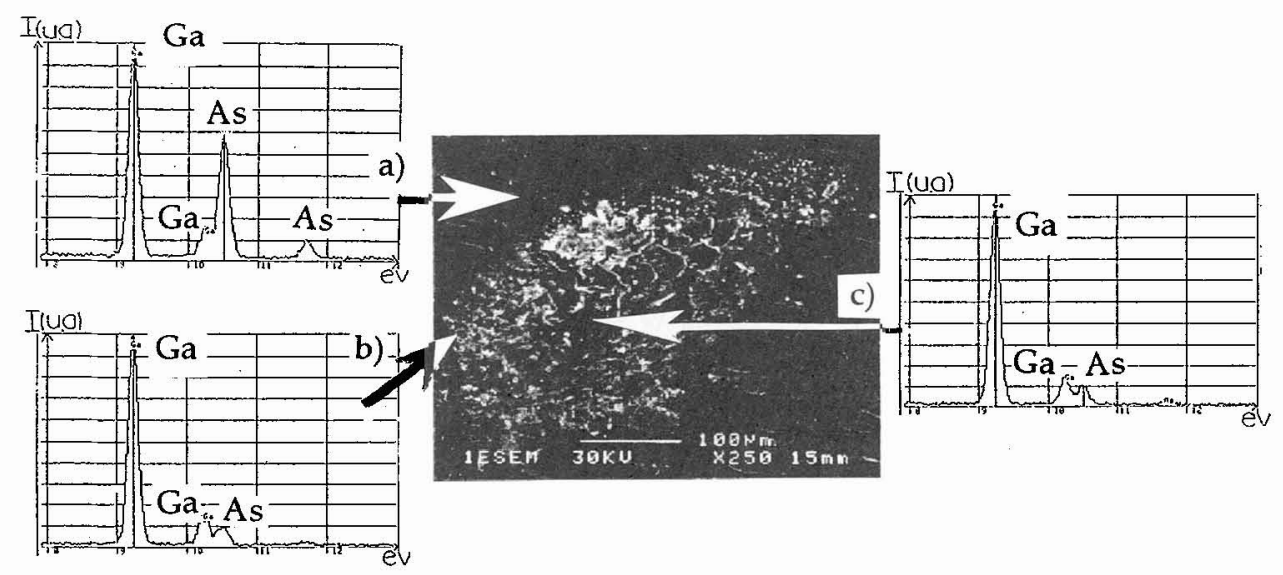

Fig. 4 -SEM and $X$ analyses of the irradiated zone. a) virgin surface, b) droplets, c) spot centre

\section{3 - CONCLUSION}

Despite the very unusual behaviour of laser sputtering of GaAs compared to InP4 and $\mathrm{GaP}^{2}$, it appears that the interaction mechanisms are mostly thermal. The gross features of the experimental results can be interpreted from the particular thermodynamics properties of this compound which exhibits very large $\mathrm{As}_{2}$ vapour pressure above the solid as soon as the temperature exceeds the critical value. After a few laser shots, corresponding to particle emission from defect sites, the thermodynamics of GaAs appears to govern the further evolution of the laser sputtered surface.

\section{References}

1 - T. Gibert, B. Dubreuil, M.F. Barthe and J.L. Debrun, J. Appl. Phys. 74 (1993) 3506

2 - K. Hattori, A. Okano, Y. Nakai and N. Itoh, Phys. Rev. B 45 (1992) 8424

3 - M.J. Howes and D.V. Morgan, "Gallium arsenide. Material devices and circuits" (J. Wiley, 1986)

4 - B. Dubreuil, T. Gibert, M.F. Barthe and J.L. Debrun, Inst. Phys. Conf. Ser. 128 (1992) 265

5 - M. Zinke-Allmang, Surf. Sci. Report 16 (1992) 377 